\title{
FOURIER SERIES AND THE MAXIMAL OPERATOR ON THE WEIGHTED SPECIAL ATOM SPACES \\ GERALDO SOARES DE SOUZA
}

\author{
Department of Mathematics \\ Auburn University \\ Auburn University, AL 36849-5310
}

(Received November 24, 1987 and in revised form July 5, 1988)

ABSTRACT. For an interval $I$ in $[0,2 \pi]$ with halves $L$ and $R$, a weighted special atom looks like $b(t)=\frac{1}{\rho(|I|)}\left[X_{L}(t)-X_{R}(t)\right]$, where $\rho$ is a nonnegative function satisfying some properties.

We consider the weighted special atom space $B(p)$ formed by $l^{1}$ linear combinations of these weighted atoms.

We showed that if $f \in B(p)$ then its Fourier series converges almost everywhere, using the Carleson-Hunt idea on their famous result about the almost everywhere convergence on $L_{p}$-spaces.

KEY WORDS AND PHRASES. Maximal operator, Lorentz Spaces and Fourier Series. 1980 AMS SUBJECT CLASSIFICATION CODE. Primary 42A20.

1. INTRODUCTION.

In [2] the following space was introduced. $B(p)=\left\{f:[0,2 \pi] \rightarrow R, f(t)=\sum_{n=0}^{\infty} c_{n} b_{n}(t), \sum_{n=0}^{\infty}\left|c_{n}\right|<\infty\right\}$. Each $b_{n}$ is a weighted special atom, that is, a real valued function $b$, defined on $[0,2 \pi]$, which is either $b(t)=\frac{1}{2 \pi}$ or $b(t)=\frac{1}{\rho(\mid I)}\left[X_{R}(t)-X_{L}(t)\right]$, where $I$ is an interval in $[0,2 \pi], L$ is the left half of $I$ and $R$ is the right half. $|I|$ denotes the length of $I, X_{E}$ the characteristic function of $E$ and $p$ is a non-negative, real valued function which is increasing, and $p(0)=0 . B(p)$ is endowed with the norm $\|f\|_{B(\rho)}=\operatorname{Inf} \sum_{n=0}^{\infty}\left|c_{n}\right|$, where the infimum is taken over all possible representations of $f . B(\rho)$ is a Banach space. For more details about this space $B(p)$ the reader is referred to [2].

The Carleson-Hunt theorem on the almost everywhere convergence of a function $f$ in $L_{p}$ for $1<p<\infty$, asserts that if $f \in L_{p}$, then the Fourier series of $f$, denoted by $S(f, x)$, converges to $f$ almost everywhere. (See [1] and [5].) In this note, using basically the idea of Carleson-Hunt, we will prove that with some additional condition on $\rho$ if $f \varepsilon B(\rho)$ then the operator 
$T f(x)=\sup _{n}\left|S_{n}(f, x)\right|$ where $S_{n}(f, x)$ is the $n t h$ partial sum of the Fourier series of $f$, is bounded into some Banach space $L(\phi)$.

We would like to point out that other direct proofs of the almost everywhere convergence for functions in $B(p)$ are also available. For example, for some $\rho$ any function in $B(\rho)$ satisfies Dini's condition. However we prefer this one, because it is a consequence of the boundedness of the maximal operator and this boundedness could be useful in other contexts, for example in the interpolation of operators.

One of the most important features of the space $B(\rho)$ is that under some additional conditions on $\rho$, it can be identified with the space of analytic functions $F$ on the disc $D=\{z \varepsilon C ;|z|<1\}$ satisfying

$$
\int_{0}^{1} \int_{0}^{2 \pi}\left|F^{\prime}\left(r e^{i \theta}\right)\right| \frac{\rho(1-r)}{1-r} d \theta d r<\infty
$$

where the dash means the derivative of $F$ with respect to $z$. For this characterization see [2].

\section{PRELIMINARIES.}

DEFINITION 2.1. A function $\rho:(0, \infty) \rightarrow[0, \infty)$ is said to be in the class $b_{1}$ if it satisfies
i) $\rho(0)=0$,
ii) $\rho$ is non-decreasing,
iii) $\frac{\rho(t)}{t}$ is decreasing,
iv) $\int_{0}^{h} \frac{\rho(s)}{s} d s \leq C \rho(h) \quad C$ an absolute constant
v) $\int_{h}^{2 \pi} \frac{\rho(t)}{t^{2}} d t \leq c \frac{\rho(h)}{h}$ with $C$ independent of $h$ and $\rho$.

Example of functions in the class $b_{1}$ are $\rho(t)=t^{\alpha}, \rho(t)=t^{\alpha} \log \frac{2 \pi e^{1 / \alpha}}{t}$ for $0<\alpha<1$.

DEFINITION 2.2. We define the space $L(\phi)$ by $L(\phi)=\{f:[0,2 \pi] \rightarrow R$, $\left.\|f\|_{\phi}=\int_{0}^{2 \pi} f \star(t) \phi(t) d t<\infty\right\}$, where $f *$ is the decreasing rearrangement of $f$, defined by $f *(t)=\operatorname{Inf}\{y: \operatorname{meas}\{x ;|f(x)|>y\} \leq t\}$, where meas means Lebesgue measure and $\phi$ is a non-negative, decreasing function.

In [6], G. G. Lorentz showed that \|\|$_{\phi}$ is a norm if and only if $\phi$ is a non-negative, decreasing function. Also he proved that $L(\phi)$ is a Banach space.

Recall that if $S_{n}(f, x)$ is the nth partial sum of the Fourier series of $f$

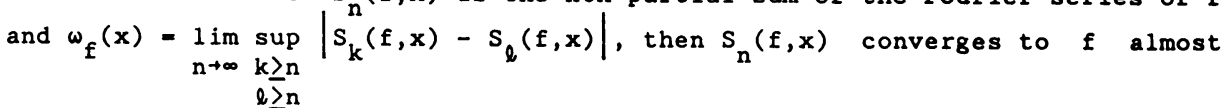
everywhere if and only if $w_{f}(x)=0$ almost everywhere.

\section{MAIN RESULT}

The main result can be stated as follows.

THEOREM. Let $\rho$ be a function in the class $b_{1}$. If $f \in B(\rho)$ then the maximal operator defined by $\operatorname{Tf}(x)=\sup _{n}\left|S_{n}(f, x)\right| \operatorname{maps} B(\rho)$ into $L(\phi)$ for $\phi(t)=\frac{\rho(t)}{t}$ boundedly, that is, $\|\mathrm{Tf}\|_{\phi} \leq M\left\|_{f}\right\|_{B(\rho)}$, where $M$ is a positive, absolute constant and $S_{n}(f, x)$ is the nth partial sum of the Fourier series of $f$. 
PROOF. First of all we notice that the operator $T_{a} f=f^{a}$ where $f^{a}(x)=f(x-a)$ maps $B(\rho)$ into $B(\rho)$ continuously. Consequently we just need to prove the result for $f_{h}(t)=\frac{1}{\rho(2 h)}\left[x_{[-h, 0)}(t)-x_{[0, h]}(t)\right], h>0$ which will follow from the estimate for $g(t)=x_{[0, h]}(t)$. In fact let $S_{n}(g, x)=\frac{1}{\pi} \int_{-\pi}^{\pi} g(t) D_{n}(x-t) d t$ where $D_{n}(t)=\frac{\sin \left(n+\frac{1}{2}\right) t}{2 \sin \frac{t}{2}}$ is the Dirichlet kernel. Therefore we have $s_{n}(f, x)=\frac{1}{\pi} \int_{x-h^{n}}^{x} D_{n}(t) d t$. We now use the elementary inequality $\left|D_{n}(t)\right| \leq \frac{C}{|t|}, 0<|t| \leq \pi$, satisfied by the Dirichlet kernels $D_{n}$, where $C$ is an absolute constant. Thus

$$
\left|s_{n}(g, x)\right| \leq \int_{x-h}^{x}\left|D_{n}(t)\right| d t \leq \int_{x-h}^{x} \frac{1}{|t|} d t \leq \frac{C h}{x-h} \leq 2 C \frac{h}{x} \text { for } x>2 h
$$

and $\left|S_{n}(g, x)\right| \leq 2 C-\frac{h}{x}$ for $x<-2 h$.

Recall that $\int_{0}^{x} D_{n}(t) d t$ is uniformly bounded in $n$ and $x$; that is, $\left|\int_{0}^{x} D_{n}(t) d t\right| \leq A$ where $A$ is an absolute constant. (See [7], volume 1, page 57.)

Consequently we have

1) $T_{g}(x) \leq A$ for all $x$

2) $\operatorname{Tg}(x) \leq 2 C \frac{h}{|x|}$ for $|x|>2 h$.

We now evaluate $\|\mathrm{Tg}\|_{\phi^{\prime}}$ for $\phi(t)=\frac{\rho(t)}{t}$. In fact, using 1) and 2) we get

$$
\begin{aligned}
\left\|T_{g}\right\|_{\phi} & =\int_{0}^{2 \pi}\left(T_{g}\right) *(x) \frac{\rho(x)}{x} d x=\int_{0}^{2 h}\left(T_{g}\right) *(x) \frac{\rho(x)}{x} d x+\int_{2 h}^{2 \pi}\left(T_{g}\right) *(x) \frac{\rho(x)}{x} d x \\
& \leq A \int_{0}^{2 h} \frac{\rho(x)}{x} d x+2 C h \int_{2 h}^{2 \pi} \frac{\rho(x)}{x^{2}} d x .
\end{aligned}
$$

Now using the conditions on $\rho$ we get

$$
\left\|\mathrm{T}_{\mathrm{B}}\right\|_{\phi} \leq \mathrm{AC} \rho(2 \mathrm{~h})+2 \mathrm{Ch} \frac{\rho(2 \mathrm{~h})}{2 \mathrm{~h}}=\operatorname{M\rho }(2 \mathrm{~h})
$$

The constant $C$ may not be the same at every occurence.

$$
\text { Consequently }\left\|T f_{h}\right\|_{\phi} \leq M \text { and so if } f(t)=\sum_{n=0}^{\infty} c_{n} b_{n}(t) \text { where } b_{n}(t)=
$$

$\frac{1}{\rho\left(\left|I_{n}\right|\right)}\left[X_{R_{n}}(t)-X_{L_{n}}(t)\right]$ and $\sum_{n=0}^{\infty}\left|c_{n}\right|<\infty$, we get $\|T f\|_{\phi} \leq M \sum_{n=0}^{\infty}\left|c_{n}\right|$ which implies $\|\mathrm{Tf}\|_{\phi} \leq M\left\|_{\mathrm{f}}\right\|_{B(p)}$. The proof is complete.

COROLLARY. Let $\rho$ be in the class $b_{1}$. If $f \in B(\rho)$, then $\left\{s_{n}(f, x)\right\}$ converges to $f(x)$ almost everywhere.

PROOF. Let $f \in B(\rho)$ then $f(x)=\sum_{n=0}^{\infty} c_{n} b_{n}(x)$ where $\sum_{n=0}^{\infty}\left|c_{n}\right|<\infty$ and the $b_{n}$ are weighted special atoms. Now observe that

$$
\begin{aligned}
w_{f}(x) & =\lim _{n \rightarrow \infty} \sup _{\substack{k>n \\
\ell>n}}\left|S_{k}(f, x)-S_{\ell}(f, x)\right| \text { satisfies } w_{f}(x)<2 T f(x) \text {. Therefore } \\
& \int_{0}^{2 \pi} w_{f}^{\star}(x) \frac{\rho(t)}{t} d t \leq 2 \int_{0}^{2 \pi}(\operatorname{Tf}(x))^{\star} \frac{\rho(t)}{t} d t \text { and so } w_{f} \varepsilon L(\phi) \text {. On the }
\end{aligned}
$$
other hand we see that $w_{f}=w_{f-f_{k}}$ where $f_{k}(x)=\sum_{n=0}^{k} c_{n} b_{n}(x)$ and 
$\left\|f_{k}-f\right\|_{B(\rho)} \rightarrow 0$ as $k \rightarrow \infty$. Then $w_{f}(x)=w_{f-f_{k}}(x) \leq 2 T\left(f-f_{k}\right)(x)$ and consequently the theorem, that is, the boundedness of $T$, implies that $\left\|w_{f}\right\|_{\phi} \leq 2\left\|T\left(f-f_{k}\right)\right\|_{\phi} \leq 2 M\left\|_{f}-f_{k}\right\|_{B(p)}$. So letting $k \rightarrow \infty$ we get $\left\|w_{f}\right\|_{\phi}=0$. Thus $w_{f}(x)=0$ almost everywhere, which implies by the comment right after definition 2.2 that $S_{n}(f, x) \rightarrow f(x)$ almost everywhere. The proof is complete. We emphasize that for $p(t)=t^{1 / p}, 1<p<\infty$ the space $B(p)$ is the space $B^{p}$ and $L(\phi)$ is the space $L(p, 1)$ in [3], and so the results in this paper on $B(\rho)$ is a generalization of that result.

We also point out that for $\rho(t)=t$, one can indeed show that there is a $f \varepsilon B(\rho)$ so that the Fourier series of $f$, diverges almost everywhere. (See [4]). This answers a question asked of the author by Professor Guido Weiss concerning almost everywhere convergence of functions in $B(\rho)$ for $\rho(t)=t$.

\section{REFERENCES}

[1] CARLESON, L., On the convergence and growth of partial sums of Fourier series, Acta. Math. 116, (1966) 135-157.

[2] BLOOM, S. and DE SOUZA, G. S., Atomic decomposition of the generalized Lipschitz spaces - To appear, Illinois Journal of Mathematics.

[3] DE SOUZA, G. S., On the convergence of Fourier series, Internat. J. Math. and Math. Sci. 7(4), (1984) 817-820.

[4] DE SOUZA, G. S. and SAMPSON, G., A function in Dirichlet space $B^{1}$ such that its Fourier series diverges almost everywhere. Preprint.

[5] HUNT, R. A., On the convergence of Fourier series, Proc. Conference Southern Illinois University, Edwardville, Ill. 1967.

[6] LORENTZ, G. G., On the theory of spaces $\Lambda$, Pacific Journal of Math. 1(1951), 411-429.

[7] ZYGMUND, A., Trigonometric Series, Cambridge University Press, London and New York, 1959.

Division of Mathematics

Foundations, Analysis and Topology

Auburn University

Auburn, AL 36849-5310 


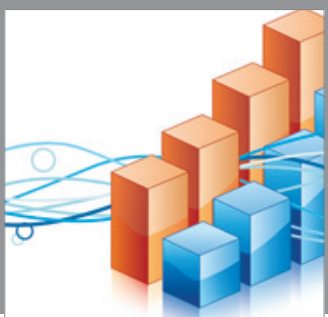

Advances in

Operations Research

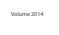



\section{The Scientific} World Journal
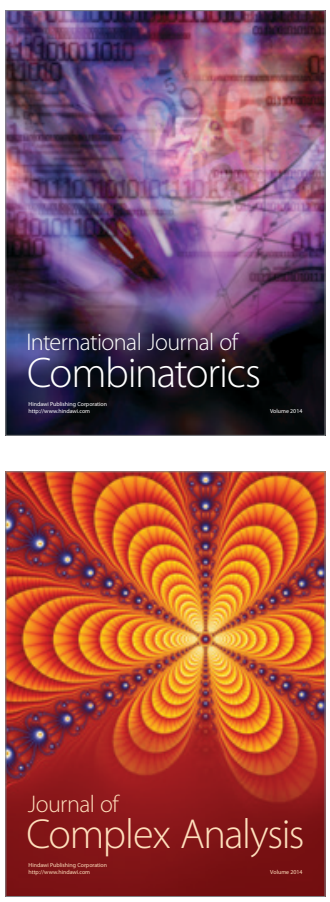

International Journal of

Mathematics and

Mathematical

Sciences


Journal of

Applied Mathematics
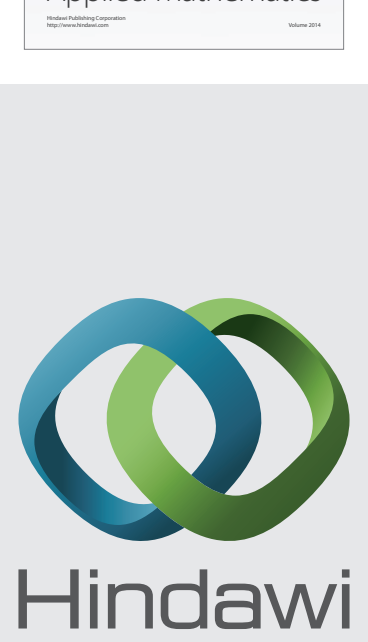

Submit your manuscripts at http://www.hindawi.com
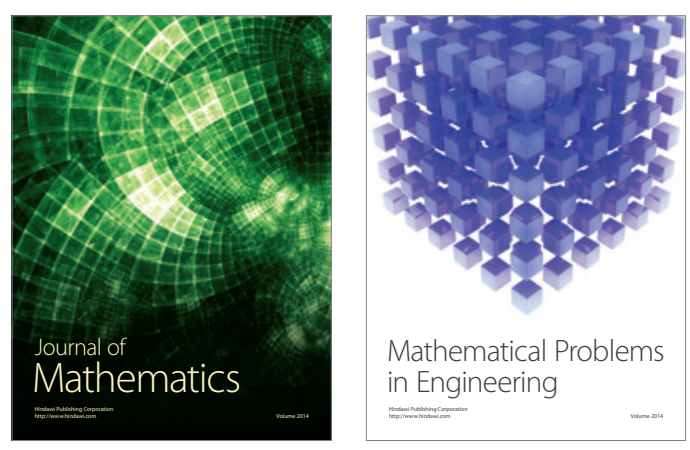

Mathematical Problems in Engineering
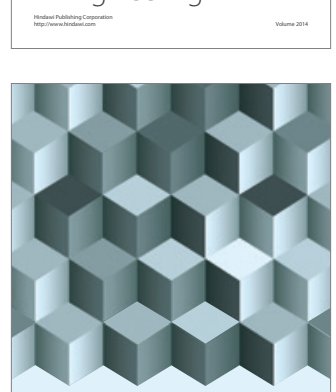

Journal of

Function Spaces
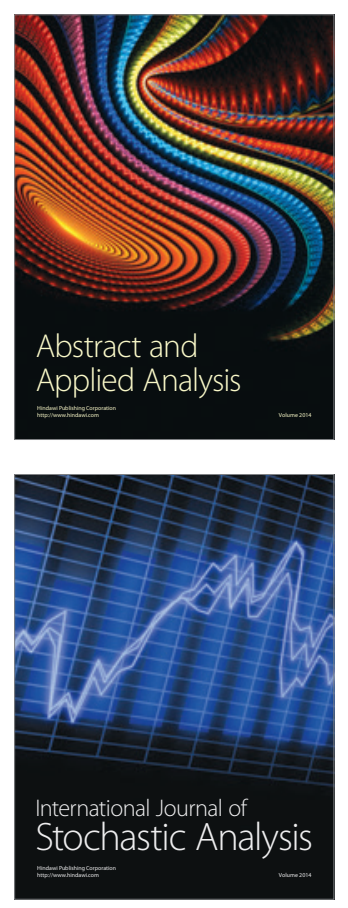

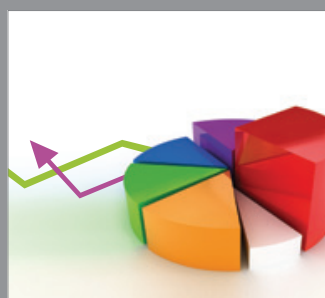

ournal of

Probability and Statistics

Promensencen
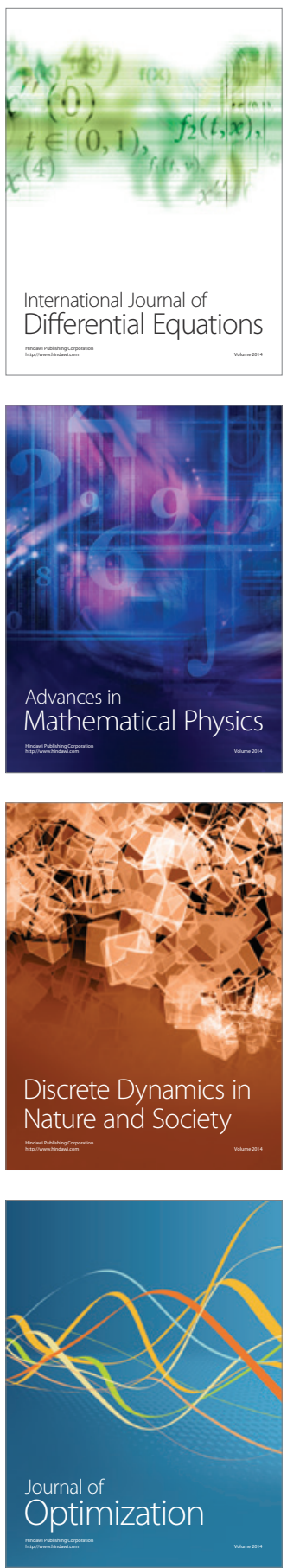